Original Research Paper

\title{
Improved 3D Imaging Performance of AFM
}

\author{
Md Sohel Rana \\ Department of Electrical and Electronic Engineering, \\ Rajshahi University of Engineering and Technology, Rajshahi, Bangladesh
}

\section{Article history}

Received: 21-12-2019

Revised: 20-01-2020

Accepted: 28-01-2020

Email: sohel@eee.ruet.ac.bd

\begin{abstract}
Over the last three decades the Atomic Force Microscopy (AFM) has played an important role in the field of nanotechnology for imaging and manipulating samples. However, the acquisition of a highquality imaging performance by an AFM is significantly influenced by its key scanning element called its Piezoelectric Tube Actuator (PTA). In this article, to improve its performance, a multi-input multi-output model predictive control scheme for achieving improved 3D scanned image of sample is proposed. The proposed controller achieves this by greatly overcoming the problem of tilted characters and nonlinearity effects in the AFM scanned images. The experimental outcomes are demonstrating the effectiveness of the proposed control technique.
\end{abstract}

Keywords: Atomic Force Microscope (AFM), Piezoelectric Tube Actuator (PTA), Nonlinearities, Multi-Input Multi-Output (MIMO), Model Predictive Control (MPC)

\section{Introduction}

Over recent years, Atomic Force Microscopy (AFM) has proven to be exceedingly handy as an investigative and manipulative research tool in the field of nanotechnology (Binnig et al., 1986). It has significant impacts in many disciplines, from biological and medical research to surface science and has almost all the capabilities required to make it a preferred imaging technique (Rana et al., 2017). It can obtain the high precision and high-resolution topographical images both in the form of 2D and 3D. Figure 1 shows a 3D scanned image of a TGQ1 sample for a scanning speed of $1 \mathrm{~Hz}$ by using AFM.

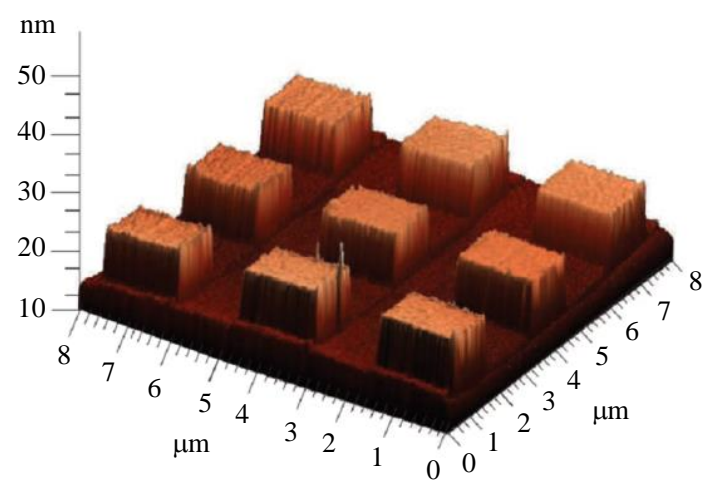

Fig. 1: 3D form of the TGQ1 sample
Nevertheless, at high scanning speed the imaging performances of presently available AFMs are constrained by the limitations of their scanning element called Piezoelectric Tube Actuator (PTA) (Li et al., 2019 and Rana et al., 2018). The major limitations of the AFM's PTA are the non-linear manners in the form of hysteresis (Zhang et al., 2019) and creep effects (Rana et al., 2014a); low resonance frequency (Moheimani and Vautier, 2005) and motion coupling effect among the axes (Rana et al., 2014b).

To achieve the high-speed AFM imaging control techniques are needed, to surmount the aforementioned restrictions of the PTA. At low scanning speed the AFM's in-built Proportional-Integral (PI) controller achieves good tracking of the reference signal. Nevertheless, at high scanning rate it cannot compensate the PTA nonlinearities. Also, it fails to damp the dominant mode of the PTA which consequences vibrations in a scanned image. During the last three decades, different control techniques have been applied to the PTA to improve the imaging performance of the AFM.

To compensate the hysteresis effect in an AFM's PTA different advanced level controllers have been addressed in the literature, such as the Iterative Learning Control (ILC) (Leang and Devasia, 2006) and $H_{\infty}$ control (Chuang et al., 2013). Leang and Devasia (2006) an improved controller is designed using a complex Preisach hysteresis model to compensate the nonlinear hysteresis effect in the PTA. Though this controller compensates the 
hysteresis effect significantly it produces vibrations in the scanned images at higher scanning rates because it cannot take effective measures over the dominant mode. An integrated inversion-based control approach is addressed in (Croft et al., 2001) to reduce the effect of creep effect.

To damp the dominant mode of the AFM's PTA numerous feedback controllers have been applied. In (Mahmood and Moheimani, 2009) the Positive Position Feedback (PPF) control scheme is proposed. It is a low-pass filter with high gain at low frequencies and fast roll-off at high frequencies. However, it suffers from the crisis of small phase and gain margin due to its low-pass nature.

One of the major limitations in AFM imaging is the motion coupling effect among the axes of its scanning unit. In the high-speed precision positioning of the PTA the motion coupling effect between its axes initiates a noteworthy amount of error. Because of this effect, the input applied to any of the axes of a PTA results in movements in both axes of the scanner and introduces errors on scanned images. In (Wu et al., 2009) an Inversion-based Iterative Control (IIC) method is proposed to compensate the motion coupling effect of a PTA in the tapping-mode AFM imaging. Although the performance of this controller is restricted up to $24.4 \mathrm{~Hz}$ scanning speed.
Also, developed mechanical structure of the PTA can overcome for its motion coupling effect. A XY nanopositioning stage actuated with piezoelectric material is presented in (Yong et al., 2009) which extensively reduces the motion coupling effect. Its combined nature with feedforward control and Integral Resonant Control (IRC) achieved high-speed scans up to $400 \mathrm{~Hz}$.

In order to overcome these existing problems, this article describes the use of an improved Model Predictive Control (MPC) scheme in a Multi-Input Multi-Output (MIMO) form. To track the reference trajectories and, compensate the nonlinearities and the motion coupling effect of the PTA is the primary goal of this control framework.

\section{Indentified Model of the PTA}

In nanopositioning applications the PTA is the most useful actuator to perform the displacement of the object in both 2D and 3D plane. Figure 2 shows the block diagram of the experimental platform. In this experiment, the plant model is developed using the black box system identification method. The best fit model frequency responses for the $X$ and $Y$-piezos are shown in Fig. 3 .

By the following frequency response functions the lateral displacements of the PTA can be described:

$$
\begin{aligned}
& G_{x x}=\frac{-1.197 \times 10^{4} s^{3}-2.289 \times 10^{6} s^{2}+1.205 \times 10^{9} s-1.599 \times 10^{13}}{s^{4}+3.859 \times 10^{5} s^{3}+6.626 \times 10^{7} s^{2}+1.806 \times 10^{11} s+2.849 \times 10^{13}} \\
& G_{x y}=\frac{4.242 s^{3}-2460 s^{2}+2.682 \times 10^{6} s-8.622 \times 10^{8}}{s^{4}+95.6 s^{3}+1.016 \times 10^{6} s^{2}+4.498 \times 10^{7} s+2.56 \times 10^{11}} \\
& G_{y x}=\frac{0.9309 s^{3}-5498 s^{2}+2.551 \times 10^{6} s-2.692 \times 10^{9}}{s^{4}+379.8 s^{3}+9.67 \times 10^{5} s^{2}+1.772 \times 10^{8} s+2.327 \times 10^{11}} \\
& G_{y y}=\frac{-40.86 s^{3}+2.703 \times 10^{4} s^{2}-1.363 \times 10^{7} s-6.895 \times 10^{10}}{s^{4}+1.763 \times 10^{3} s^{3}+7.812 \times 10^{5} s^{2}+8.214 \times 10^{8} s+1.316 \times 10^{11}}
\end{aligned}
$$

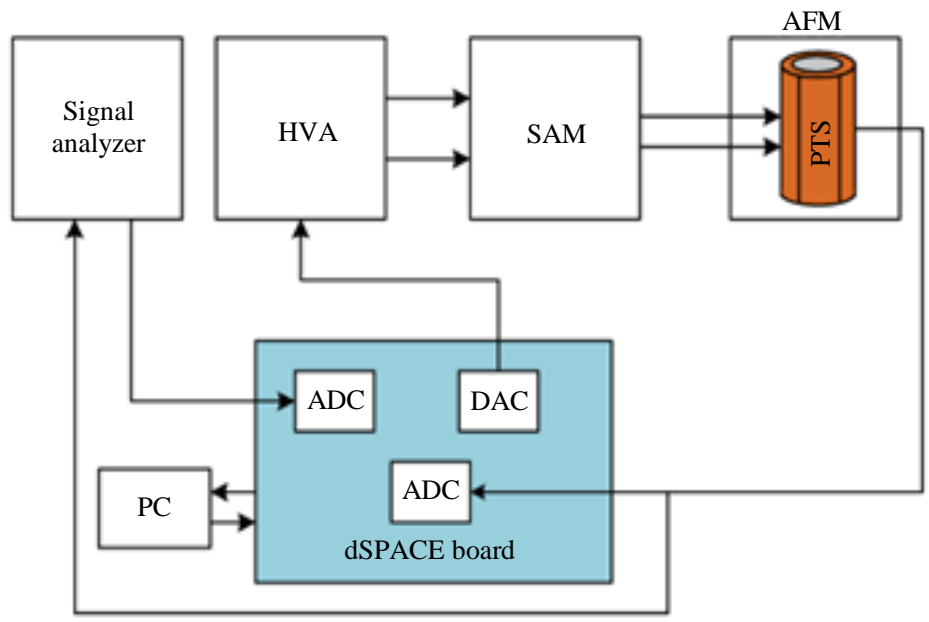

Fig. 2: Schematic diagram of the experimental platform 

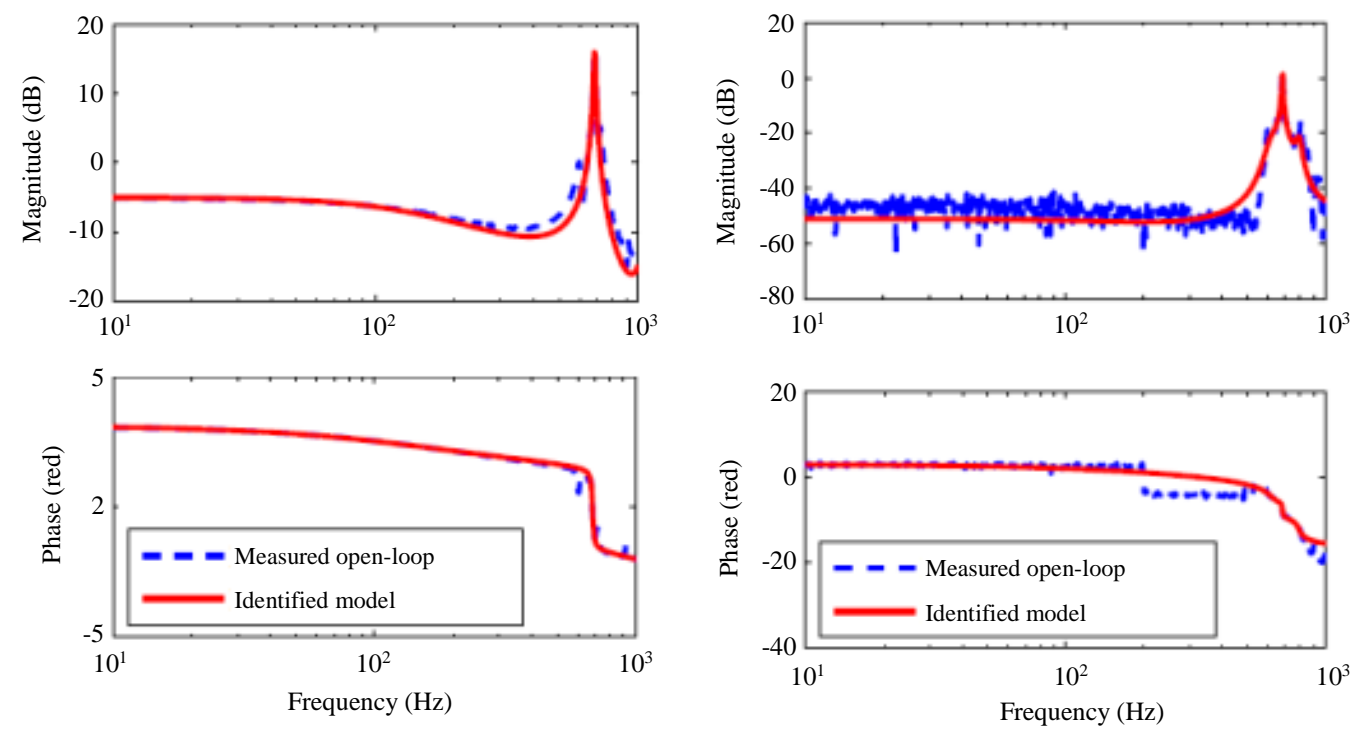

(a)

(b)
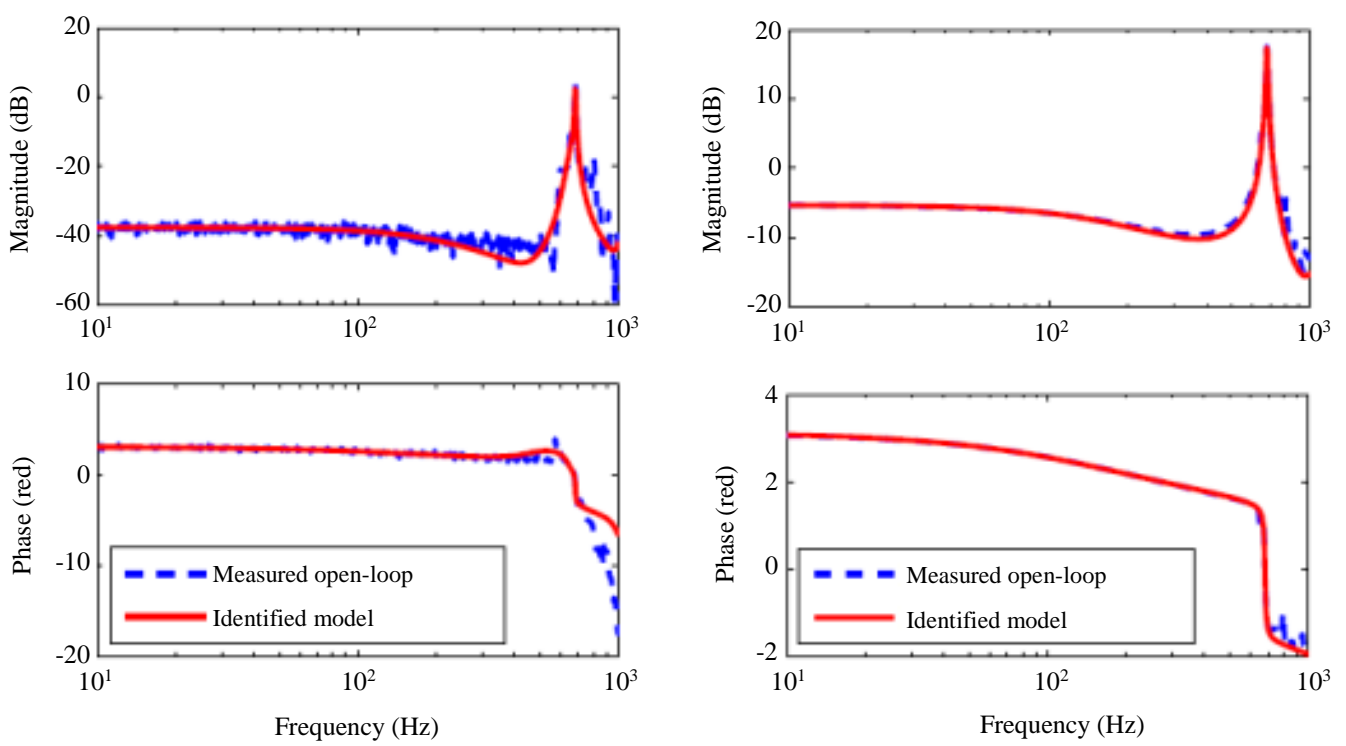

(c)

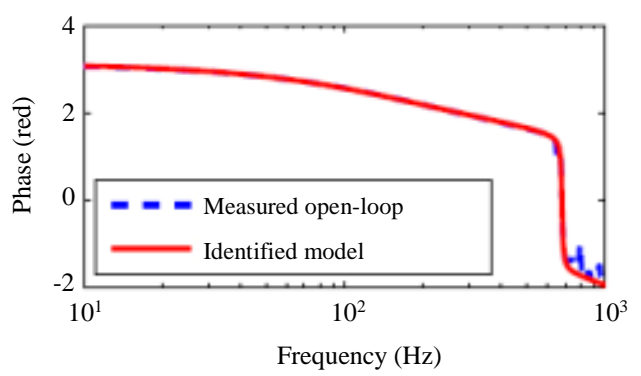

(d)

Fig. 3: The measured and identified model of the PTA's dynamics: (a) $G_{x x}$, (b) $G_{x y}$, (c) $G_{y x}$ and (d) $G_{y y}$ [measured system model (dashed line - -) and identified system model (solid line -)] (Rana et al., 2014a)

\section{Design of Controller}

\section{Design of PI Controller}

The design of a PI controller is presented in this section. It continuously observes an inaccuracy between a preferred reference and a measured value then applies an improvement to the plant based on action of proportional and integral control. The generalized transfer function of the PI controller is as follows:

$G_{c}=K_{P}+\frac{K_{I}}{s}$
A detail design of the PI controller can be found in (Mandava and Vundavilli, 2019; Mandava and Vundavilli, 2018).

\section{Design of Damping Compensator Controller}

The design of a Damping Compensator (DC) controller is presented in this section. The form of the DC controller is (Rana et al., 2014c):

$M_{q}(s)=\sum_{q=1}^{N}-k_{q} \frac{C_{q} s\left(R_{q}+L_{q} s\right)}{L_{q} C_{q} s^{2}+R_{q} C_{q} s+1} ;$ 
where, $q=1,2, \cdots, N, k_{q}$ is the controller gain of the resultant mode. We are able to get better damping of the dominant mode of the PTA by choosing the correct value of $L_{q}, R_{q}$ and $C_{q}$. In this work the DC controller is implemented with the PI controller.

\section{Design of MIMO MPC Controller}

There are numerous potential applications for MPC schemes, such as biomedical control, MEMS, robot manipulation, automotive or aerospace control, power electronics, microchemical systems and different process industries. Their good performances demonstrate their capability to achieve highly efficient control in the field of high- speed AFM imaging. They have some special features which make them useful in this area, such as tracking a reference signal, having a high bandwidth, handling time delays and constraints, augmenting integral actions and possessing a multivariable nature. The MIMO form of a MPC controller improves the overall stability of the system. These capabilities of MPC have motivated this research to indirectly compensate the limitations in an AFM's PTA. In this control design, the dynamics of the PTA is expressed as the following discrete time-domain model (Rana et al., 2014a):

$x_{p}(k+1)=A_{p} x_{p}(k)+B_{p} u(k)$

$y(k)=C_{p} x_{p}(k)$

where, $A_{\mathrm{p}}, B_{\mathrm{p}}$ and $C_{\mathrm{p}}$ describe the plant dynamics, derived at a sampling time $T_{s}$ from Equations (1)-(4). To track the reference signal and to compensate the system nonlinearities an integrator is added in MPC algorithm:

$$
\begin{aligned}
& {\left[\begin{array}{c}
\Delta x_{p}(y+1) \\
y(k+1)
\end{array}\right]=A_{C}\left[\begin{array}{c}
\Delta x_{p}(k) \\
y(k)
\end{array}\right]+B_{C} \Delta u(k) ;} \\
& y(k)=C_{C}\left[\begin{array}{c}
\Delta x_{p}(k) \\
y(k)
\end{array}\right] ;
\end{aligned}
$$

where:

$$
A_{C}=\left[\begin{array}{cc}
A_{p} & 0 \\
C_{p} A_{p} & I
\end{array}\right] ; \quad B_{C}=\left[\begin{array}{c}
B_{p} \\
C_{p} B_{p}
\end{array}\right] ; \quad C_{C}=\left[\begin{array}{ll}
0 & I
\end{array}\right] ;
$$

where, $A_{C}, B_{C}$ and $C_{C}$ are the augmented plant model. The following cost function is minimized to develop the proposed control law:

$$
\begin{aligned}
& J=\sum_{m=1}^{N_{p}} \bar{Q}\left(y(k+m \mid k)-R_{s}(k+m)\right)^{2} \\
& +\sum_{m=1}^{N_{c}} \bar{R}(\Delta u(k+m-1))^{2} ;
\end{aligned}
$$

where the linear inequality constraints on the system inputs are:

$$
\begin{aligned}
& u_{\text {min }} \leq u(k+i-1) \leq u_{\text {max }}, \quad i=1, \cdots, N_{c} ; \\
& \Delta u_{\text {min }} \leq \Delta u(k+i-1) \leq \Delta u_{\max }, \quad i=1, \cdots, N_{c} ;
\end{aligned}
$$

where, $R_{S}, \bar{R}$ and $\bar{Q}$ are the reference signal, the control weighting matrix and the state weighting matrix, respectively.

\section{Experimental Results}

Figure 4 and 5 shows the scanned 3D images of a sample and its vertical direction contours at $62.50 \mathrm{~Hz}$ and $125 \mathrm{~Hz}$ scanning speeds. The AFM is controlled in constant force mode for the period of this experiment. As a reference sample in this experiment an NT-MDT TGQ1 is used. The scanning is performed using an NT-MDT CSG01 microcantilever. The shape of TGQ1 sample is rectangular whose altitude is $20 \pm 1.5 \mathrm{~nm}$ and area is $1.5 \pm 0.25 \mu \mathrm{m}$. The conventional zigzag raster pattern scanning technique is used in this experimental investigation.

\section{Discussion}

To evaluate the nanopositioning performance of the proposed controller it is implemented in the AFM's PTA. At $62.50 \mathrm{~Hz}$ scanning speed the scanned 3D images using the conventional PI controller and DC controller have some noticeable error at the boundary of the image. This consequence is excessive at high scanning rate. At $125 \mathrm{~Hz}$ scanning speed the DC controller has alleviated this error in some degree where the PI controller entirely looses the information of the image because of the nonlinear effects and excitation of resonance of the AFM's PTA. On the contrary, the scanned 3D images using the proposed controller are better capture, even at high speeds. In Fig. 5 the vertical contours of the scanned images are presented. It can be noticed that the proposed control scheme provide the better height profile of the calibration grating than the existing AFM PI controller and the DC controller which results in improved images. 


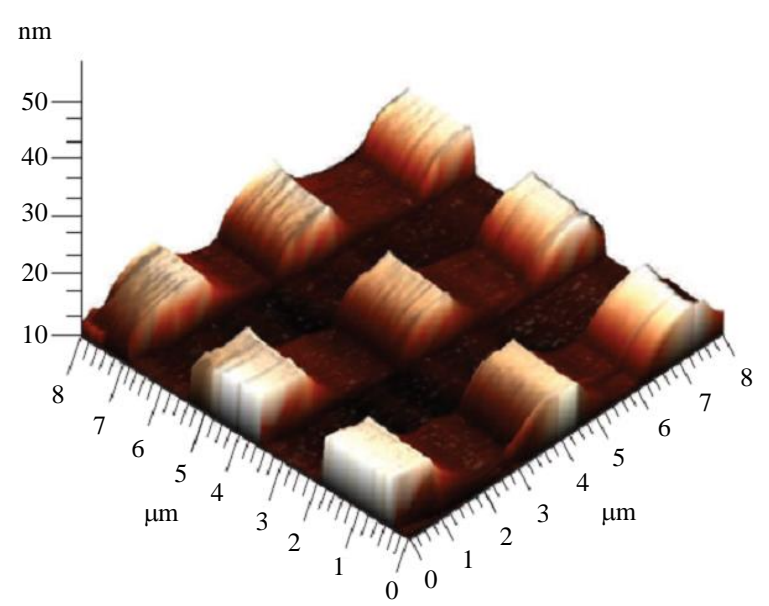

(a)

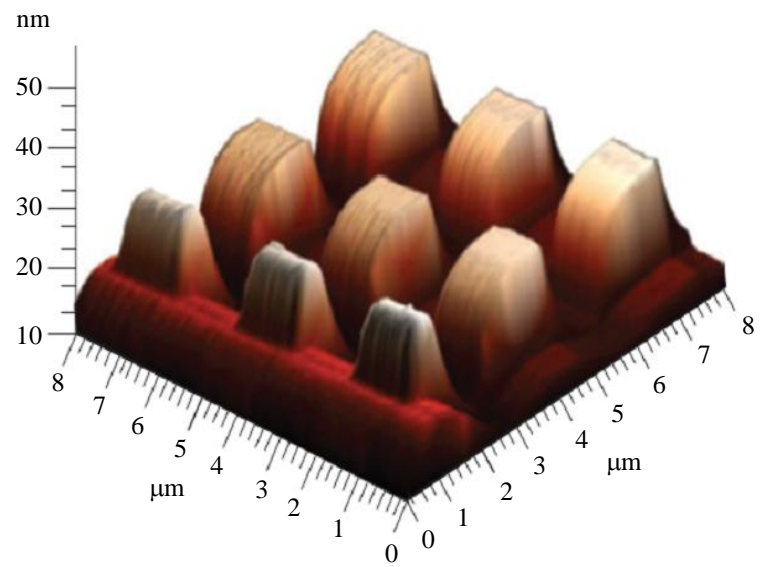

(c)

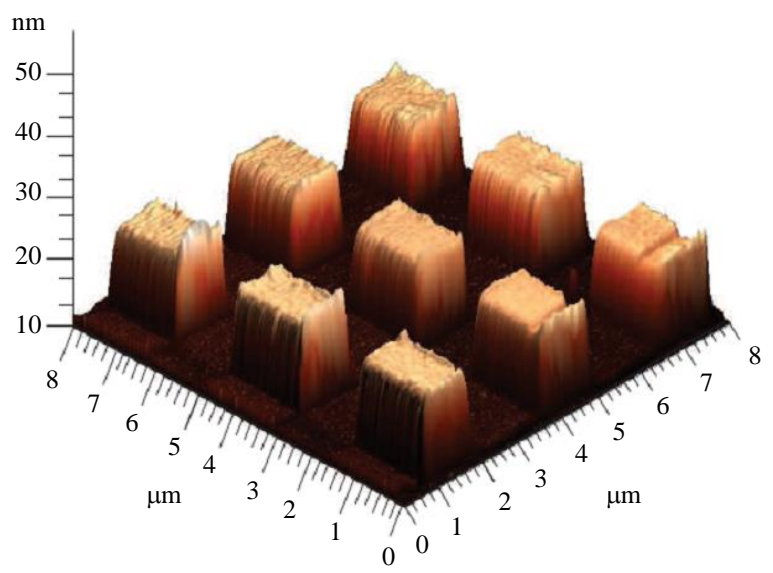

(e)



(b)

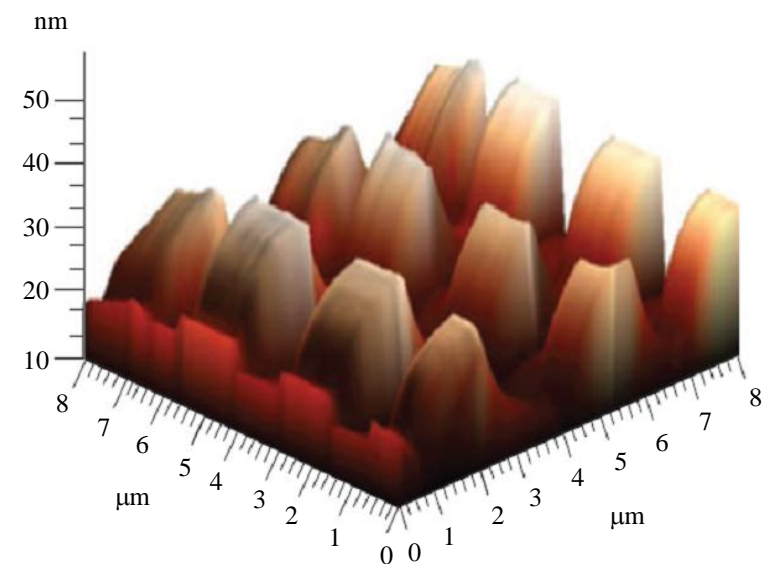

(d)



(f)

Fig. 4: 3D scanned images at 62.50 and $125 \mathrm{~Hz}$ : (a)-(b) using the PI controller; (c)-(d) using the DC controller; and (e)-(f) using the proposed controller, respectively 


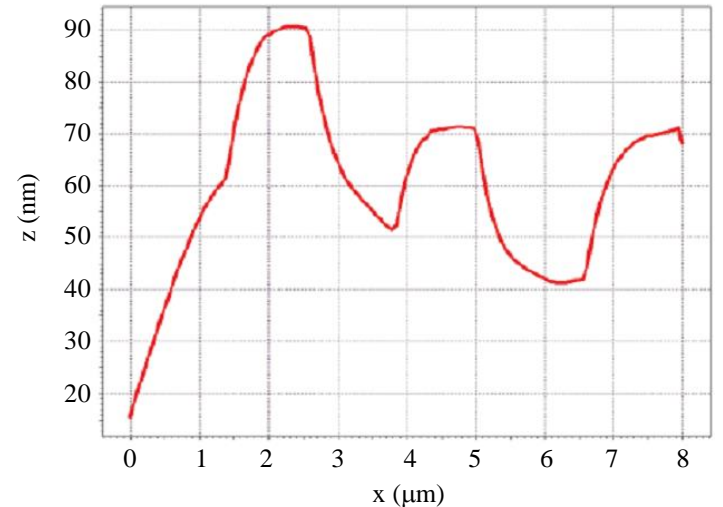

(a) $62.50 \mathrm{~Hz}$

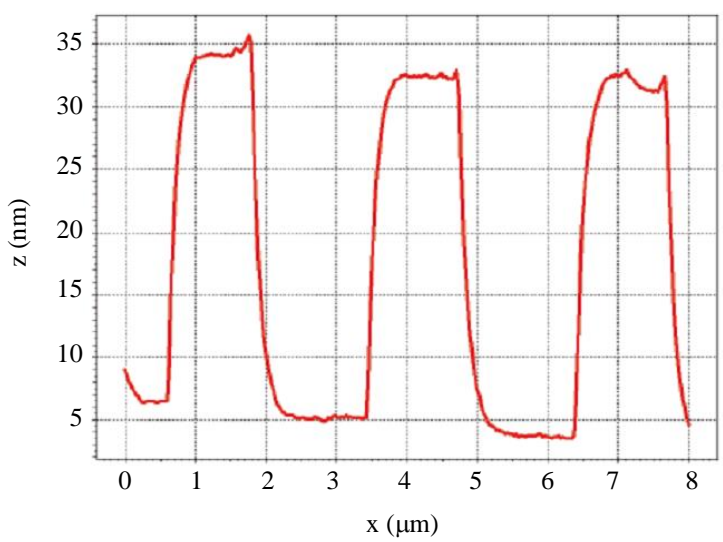

(c) $62.50 \mathrm{~Hz}$

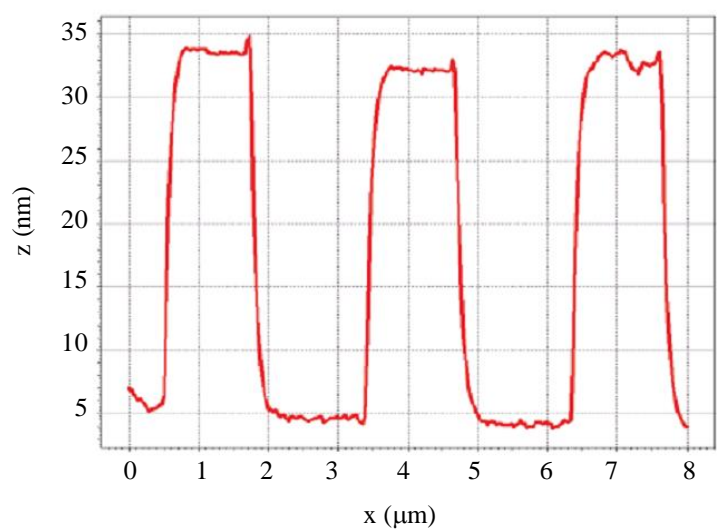

(e) $62.50 \mathrm{~Hz}$

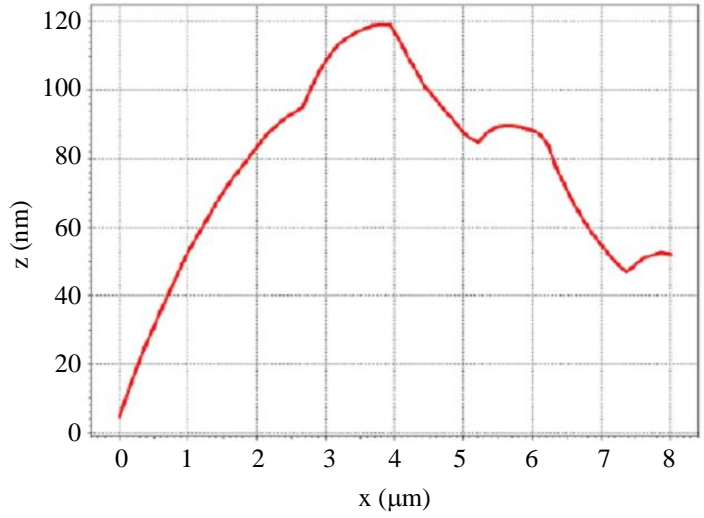

(b) $125 \mathrm{~Hz}$

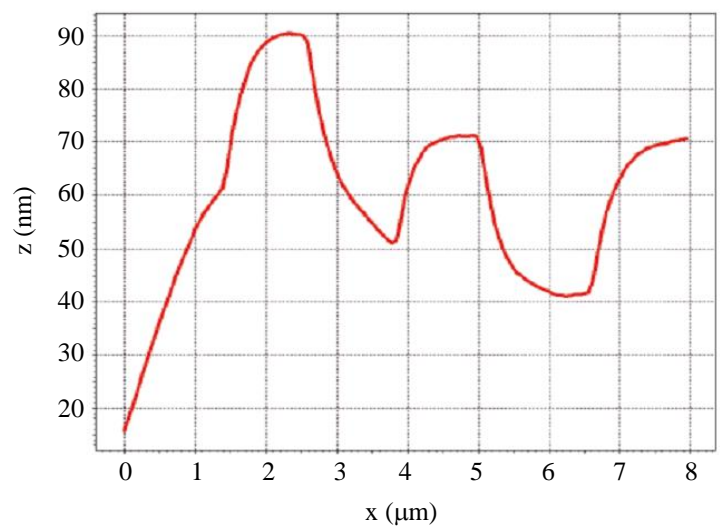

(d) $125 \mathrm{~Hz}$

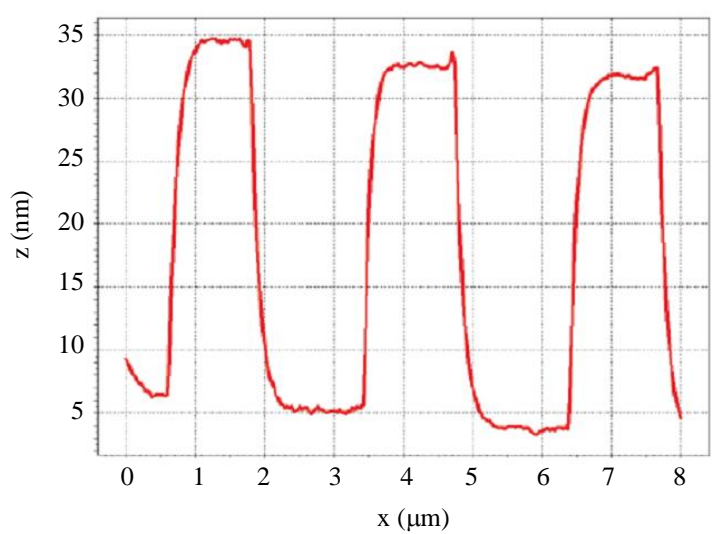

(f) $125 \mathrm{~Hz}$

Fig. 5: Vertical direction contours of the sample at 62.50 and $125 \mathrm{~Hz}$ : (a)-(b) using the PI controller; (c)-(d) using the DC controller; and (e)-(f) using the proposed controller, respectively

\section{Conclusion and Future Work}

In this brief, the design and experimental execution of a MIMO MPC controller for advancing the 3D imaging performance of an AFM is addressed. The shortcomings of the PTA are compensated by the proposed controller. The proposed controller results improved 3D scanned images over the existing PI controller and the DC controller up to $125 \mathrm{~Hz}$ scanning speed.

In this work to generate the 3D scanned images of the sample a conventional scanning method is used which excites tube resonance. In future, a Lissajous scanning pattern could be a better alternative. 


\section{Acknowledgement}

A special thanks goes to Mr. Shane Brandon, the UNSW, Canberra, ACT-2620, Australia for his important suggestions at some stage in the experimental investigations.

\section{Ethics}

This article is an original research paper. There are no ethical issues that may arise after the publication of this namuscript.

\section{References}

Binnig, G.K., C.F. Quate and C. Gerber, 1986. Atomic Force Microscope (AFM). Phys. Rev. Lett., 56: 930-933. DOI: 10.1103/PhysRevLett.56.930

Chuang, N., I.R. Petersen and H.R. Pota, 2013. Robust $H_{\infty}$ control in fast atomic force microscopy. Asian J. Control, 15: 1-15. DOI: 10.1002/asjc.585

Croft, D., G. Shedd and S. Devasia, 2001. Creep, hysteresis and vibration compensation for piezoactuators: Atomic force microscopy application. Trans. ASME J. Dyn. Syst. Meas. Control, 123: 35-43. DOI: 10.1115/1.1341197

Leang, K.K. and S. Devasia, 2006. Design of hysteresis compensating iterative learning control for piezopositioners: Application to atomic force microscopes. Mechatronics, 16: 141-158.

DOI: 10.1016/j.mechatronics.2005.11.006

Li, L., C. Li, G. Gu and L. Zhu, 2019. Modified repetitive control based cross-coupling compensation approach for the piezoelectric tube scanner of atomic force microscopes. IEEE/ASME Trans. Mechatron., 24: 666-676.

DOI: $10.1109 / \mathrm{TMECH} .2019 .2893628$

Mahmood, I.A. and S.O.R. Moheimani, 2009. Making a commercial atomic force microscope more accurate and faster using positive position feedback control. Rev. Sci. Instrum., 80: 063705-063708.

DOI: $10.1063 / 1.3155790$

Mandava, R.K. and P.R. Vundavilli, 2018. Implementation of modified chaotic invasive weed optimization algorithm for optimizing the PID controller of the biped robot. Sādhanā, 43: 66-66. DOI: $10.1007 / \mathrm{s} 12046-018-0851-9$

Mandava, R.K. and P.R. Vundavilli, 2019. An optimal PID controller for a biped robot walking on flat terrain using MCIWO algorithms. Evol. Intel., 12: 33-48. DOI: 10.1007/s12065-018-0184-y

Moheimani, S.O.R. and B.J.G. Vautier, 2005. Resonant control of structural vibration using charge-driven piezoelectric actuators. IEEE Trans. Control Syst. Technol., 13: 1021-1035.

DOI: $10.1109 / \mathrm{TCST} .2005 .857407$
Rana, M.S., H.R. Pota and I.R. Petersen, 2014a. Creep, hysteresis and vibration effects attenuation in an AFM PTA. Proceedings of the European Control Conference, Jun. 24-27, IEEE Xplore Press, Strasbourg, France, pp: 2022-2027. DOI: 10.1109/ECC.2014.6862417

Rana, M.S., H.R. Pota and I.R. Petersen, 2014b. Crosscoupling effect compensation of an AFM piezoelectric tube scanner for improved nanopositioning. Proceedings of the American Control Conference, Jun. 4-6, IEEE Xplore Press, Portland, Oregon, USA, pp: 2456-2461.

DOI: $10.1109 / A C C .2014 .6859357$

Rana, M.S., H.R. Pota and I.R. Petersen, 2014c. Spiral scanning with improved control for faster imaging of AFM. IEEE Trans. Nanotechnol., 13: 541-550. DOI: 10.1109/TNANO.2014.2309653

Rana, M.S., H.R. Pota and I.R. Petersen, 2017. Improvement in the imaging performance of atomic force microscopy: A survey. IEEE Trans. Automat. Sci. Eng., 14: 1265-1285. DOI: $10.1109 /$ TASE.2016.2538319

Rana, M.S., H.R. Pota and I.R. Petersen, 2018. A survey of methods used to control piezoelectric tube scanners in high-speed AFM imaging. Asian J. Control, 20: 1379-1399. DOI: 10.1002/asjc.1728

Wu, Y., J. Shi, C. Su and Q. Zou, 2009. A control approach to cross-coupling compensation of piezotube scanners in tapping-mode atomic force microscope imaging. Rev. Sci. Instrum., 80: 043709-0437010. DOI: 10.1063/1.3124183

Yong, Y., S. Aphale and S.O.R. Moheimani, 2009. Design, identification and control of a flexure-based $\mathrm{XY}$ stage for fast nanoscale positioning. IEEE Trans. Nanotechnol., 8: 46-54. DOI: 10.1109/TNANO.2008.2005829

Zhang, L., X. Chen, J. Huang, H. Li and L. Chen et al., 2019. A method to correct hysteresis of scanning probe microscope images based on a sinusoidal model. Rev. Sci. Instrum., 90: 023704-023708. DOI: $10.1063 / 1.5052281$ 